\title{
Kerriamycin B inhibits protein SUMOylation
}

\author{
Isao Fukuda ${ }^{1,2}$, Akihiro Ito ${ }^{1,3,4}$, Masakazu Uramoto ${ }^{5,6}$, Hisato Saitoh ${ }^{7}$, Hisashi Kawasaki ${ }^{8}$, Hiroyuki Osada ${ }^{2,5,6}$ \\ and Minoru Yoshida ${ }^{1,2,3,4}$
}

The Journal of Antibiotics (2009) 62, 221-224; doi:10.1038/ja.2009.10; published online 6 March 2009

Keywords: kerriamycin B; SUMO-1; SUMO-activating enzyme (E1)

Post-translational conjugation of small ubiquitin-related modifier protein (SUMO) to protein substrates (SUMOylation) has been revealed as one of the major post-translational regulatory systems in animals and other eukaryotes. SUMO conjugation is catalyzed by a multi-step enzymatic reaction cascade similar to ubiquitinylation. ${ }^{1}$ In the first step, the SUMO precursor is cleaved near the C-terminus by SUMO-specific proteases to expose a C-terminal diglycine. The C-terminal glycine of mature SUMO then forms a thioester linkage to the cysteine residue of SUMO-activating enzyme (E1), the Aos1/Uba2 heterodimer, to generate the E1-SUMO intermediate in an ATP-dependent manner. Next, SUMO is transferred to the active site of the cysteine residue of the SUMOconjugating enzyme (E2), Ubc9, through another thioester bond. In the last step, E2 and the SUMO ligase (E3) catalyze SUMOylation of substrate proteins at the $\varepsilon$-amino group of internal lysine residues. Although enzymatic reactions by E1 and E2 are sufficient for catalyzing in vitro SUMOylation in most cases, E3s facilitate both in vivo and in vitro conjugation and are important for substrate specificity. ${ }^{1}$

The structure of SUMO is similar to that of ubiquitin, but its functions are different. SUMOylation regulates protein subcellular localization, enzymatic activity and protein stability, which are associated with the cell cycle, transcription, DNA repair and innate immunity. ${ }^{2,3}$ In addition, SUMOylation has been recently linked causally to diseases, such as Alzheimer's and Huntington's diseases, ${ }^{4}$ viral infection ${ }^{5}$ and cancer. ${ }^{6,7}$ Notwithstanding the importance of SUMOylation in regulating diverse life phenomena and diseases, small molecule inhibitors of SUMOylation have been unexplored. Here, we report novel activity of kerriamycin B that inhibits protein SUMOylation, which will provide useful information about the role of SUMOylation in cells and drug development.

\section{MATERIALS AND METHODS}

Materials

Kerriamycin B was purified from a culture broth of an unidentified strain of actinomycetes. A goat polyclonal anti-SUMO-1 (N-19) antibody was purchased from Santa Cruz Biotechnologies (Santa Cruz, CA, USA). A mouse monoclonal anti-T7 antibody was from Novagen (Darmstadt, Germany). Mouse monoclonal anti- $\alpha$-tubulin (B-5-1-2) and anti-FLAG (M2) antibodies were purchased from Sigma (St Louis, MO, USA). Recombinant His and T7-tagged RanGAP1-C2, GST-Aos1-Uba2 fusion protein (E1), His-tagged Ubc9 (E2) and His-tagged SUMO-1 proteins were purified as previously described. ${ }^{8}$

\section{Isolation of kerriamycin B}

After extraction of kerriamycin B from the 4-day cultured broth filtrate with $50 \mathrm{ml}$ of $n-\mathrm{BuOH}$, the crude material was partitioned between water and EtOAc. After removal of EtOAc from the organic layer, the extract was dissolved in $\mathrm{MeOH}$ and subjected to ODS open column chromatography. Active substance $(2 \mathrm{mg})$ was given by concentration of the fraction eluted by $80 \%$ aq. MeOH. Further purification was carried out by using preparative HPLC (Waters 600, column (Waters, Milford, MA, USA); PEGASIL ODS $20 \times 250 \mathrm{~mm}$, monitor (Senshu Scientific, Tokyo, Japan); $220 \mathrm{~nm}$, mobile phase; $30 \%$ aq. $\left.\mathrm{CH}_{3} \mathrm{CN}\right)$. The active eluate at $28 \mathrm{~min}$ was collected to give a yellow powder $(1.6 \mathrm{mg})$. HR-ESI-MS m z ${ }^{-1} 843.34507[\mathrm{M}-\mathrm{H}]^{-}(843.34392$ calculated for $\mathrm{C}_{43} \mathrm{H}_{55} \mathrm{O}_{17}$ ); UV $\lambda_{\max }(\mathrm{MeOH}) \mathrm{nm}(\varepsilon) 296.0$ (sh, 5595), 320.0 (4895), 422.0 (5038); $[\alpha]_{589}{ }^{22}+11.4^{\circ}(\mathrm{c} 0.084, \mathrm{MeOH}) ;{ }^{1} \mathrm{H}-\mathrm{NMR}\left(600 \mathrm{MHz}, \mathrm{CD}_{3} \mathrm{OD}\right) \delta_{\mathrm{H}}$ (p.p.m., $J$ value $\fallingdotseq \mathrm{Hz}): \overline{7} .8(\mathrm{~d}, J=7.9,10-\mathrm{H}), 7.5(\mathrm{~d}, J=7.2,11-\mathrm{H}), 6.89(\mathrm{~d}$, $J=9.7,6-\mathrm{H}), 6.39$ (d, $J=9.3,5-\mathrm{H}), 5.27$ (brs, $J=2.0,1^{\prime \prime \prime}-\mathrm{H}$ ), 4.96 (brs, $J=2>$, $\left.1^{\prime \prime}-\mathrm{H}\right), 4.56$ (dd, J=9.6, 1.4, $\left.1^{\prime \prime \prime \prime}-\mathrm{H}\right), 4.24$ (q, $\left.J=6.5,5^{\prime \prime}-\mathrm{H}\right), 3.78$ (ddd, $J=11.2$, $\left.8.8,4.8,3^{\prime}-\mathrm{H}\right), 3.64$ (q, J=6.7, $\left.5^{\prime \prime \prime}-\mathrm{H}\right), 3.54$ (brs, J=2>, $\left.4^{\prime \prime}-\mathrm{H}\right), 3.48$ (m, 1'-H), $3.48\left(\mathrm{~m}, 3^{\prime \prime \prime \prime}-\mathrm{H}\right), 3.48\left(\mathrm{~m}, 5^{\prime}-\mathrm{H}\right), 3.35$ (brs, $\left.4^{\prime \prime \prime}-\mathrm{H}\right), 3.21$ (dq, $J=6.2$, $\left.3.1,5^{\prime \prime \prime \prime}-\mathrm{H}\right), 3.14\left(\mathrm{~d}, J=9.0,4^{\prime}-\mathrm{H}\right), 2.89\left(\mathrm{q}, J=9.0,4^{\prime \prime \prime \prime}-\mathrm{H}\right), 2.72(\mathrm{~d}, J=13.1,2-$ Ha), 2.56 (d, $J=13.1,2-\mathrm{Hb}$ ), 2.54 (m, $2^{\prime}-\mathrm{Ha}$ ), 2.18 (ddd, $J=12.6,5.0,1.4,2^{\prime \prime \prime \prime}$ Ha), 2.07 (4-Ha), 2.06 (m, 3"'-Ha), $2.04\left(\mathrm{~m}, 3^{\prime \prime}-\mathrm{Ha}\right), 2.04$ (m, 2"-Ha), 1.98 (4$\mathrm{Hb}), 1.94\left(\mathrm{~m}, 3^{\prime \prime}-\mathrm{Hb}\right), 1.88$ (d, J=13.4, 2'"-Ha), 1.81 (d, J=13.4, 2"'-Hb), 1.58 $\left(\mathrm{m}, 3^{\prime \prime \prime}-\mathrm{Hb}\right), 1.54$ (ddd, $\left.J=12.6,5.0,1.4,2^{\prime \prime \prime \prime}-\mathrm{Hb}\right), 1.41\left(\mathrm{~m}, 2^{\prime \prime}-\mathrm{Hb}\right), 1.38$

\footnotetext{
${ }^{1}$ Chemical Genetics Laboratory, RIKEN Advanced Science Institute, Wako, Saitama, Japan; ${ }^{2}$ Graduate School of Science and Engineering, Saitama University, Saitama, Saitama, Japan; ${ }^{3}$ Chemical Genomics Research Group, RIKEN Advanced Science Institute, Wako, Saitama, Japan; ${ }^{4}$ Japan Science and Technology Corporation, CREST Research Project, Kawaguchi, Saitama, Japan; ${ }^{5}$ Antibiotics Laboratory, RIKEN Advanced Science Institute, Wako, Saitama, Japan; ${ }^{6}$ Chemical Library and Bioprobe Research Group, RIKEN Advanced Science Institute, Wako, Saitama, Japan; ${ }^{7}$ Department of Biological Sciences, Graduate School of Science and Technology, Kumamoto University, Kumamoto, Japan and ${ }^{8}$ Department of Green and Sustainable Chemistry, Tokyo Denki University, Kanda, Chiyoda-ku, Tokyo, Japan

Correspondence: Dr A Ito, Chemical Genetics Laboratory, RIKEN Advanced Science Institute, Wako, Saitama, 351-0198, Japan.

E-mail: akihiro-i@riken.jp
}

Received 15 December 2008; revised 19 January 2009; accepted 21 January 2009; published online 6 March 2009 
(d, $\left.J=5.8,6^{\prime}-\mathrm{H}\right), 1.28$ (m, 2'-Hb), $1.24\left(\mathrm{~d}, J=6.2,6^{\prime \prime \prime \prime}-\mathrm{H}\right), 1.20\left(\mathrm{~s}, 3-\mathrm{CH}_{3}\right), 1.15$ $\left(\mathrm{d}, J=6.5,6^{\prime \prime}-\mathrm{H}\right)$ and $0.51\left(\mathrm{~d}, J=6.5,6^{\prime \prime \prime}-\mathrm{H}\right) ;{ }^{13} \mathrm{C}-\mathrm{NMR}\left(150 \mathrm{MHz}, \mathrm{CD}_{3} \mathrm{OD}\right) \delta_{\mathrm{C}}$ (p.p.m.): $204.9\left(\mathrm{C}_{1}\right), 189.6\left(\mathrm{C}_{7}\right), 184.1\left(\mathrm{C}_{12}\right), 159.0\left(\mathrm{C}_{8}\right), 146.3\left(\mathrm{C}_{5}\right), 141.5$ $\left(\mathrm{C}_{12 \mathrm{a}}\right), 139.2\left(\mathrm{C}_{9}\right), 138.6\left(\mathrm{C}_{6 \mathrm{a}}\right), 134.3\left(\mathrm{C}_{10}\right), 132.4\left(\mathrm{C}_{11 \mathrm{a}}\right), 120.2\left(\mathrm{C}_{11}\right), 117.9$ $\left(\mathrm{C}_{6}\right), 115.5\left(\mathrm{C}_{7 \mathrm{a}}\right), 102.8\left(\mathrm{C}_{1^{\prime \prime \prime \prime}}\right), 95.5\left(\mathrm{C}_{1^{\prime \prime \prime}}\right), 95.2\left(\mathrm{C}_{1^{\prime \prime}}\right), 82.7\left(\mathrm{C}_{4 \mathrm{a}}\right), 82.6\left(\mathrm{C}_{12 \mathrm{~b}}\right)$, $78.4\left(\mathrm{C}_{4^{\prime \prime \prime}}\right), 77.78\left(\mathrm{C}_{5^{\prime}}\right), 77.75\left(\mathrm{C}_{3^{\prime}}\right), 77.72\left(\mathrm{C}_{4^{\prime \prime}}\right), 77.1\left(\mathrm{C}_{3}\right), 76.8\left(\mathrm{C}_{4^{\prime}}\right), 73.2$ $\left(\mathrm{C}_{5^{\prime \prime \prime \prime}}\right), 72.35\left(\mathrm{C}_{3^{\prime \prime \prime \prime}}\right), 72.32\left(\mathrm{C}_{1^{\prime}}\right), 68.1\left(\mathrm{C}_{5^{\prime \prime \prime}}\right), 67.8\left(\mathrm{C}_{4^{\prime \prime \prime}}\right), 67.7\left(\mathrm{C}_{5^{\prime \prime}}\right), 54.7\left(\mathrm{C}_{2}\right)$, $44.4\left(\mathrm{C}_{4}\right), 40.6\left(\mathrm{C}_{2^{\prime \prime \prime \prime}}\right), 37.7\left(\mathrm{C}_{2^{\prime}}\right), 29.9\left(\mathrm{C}_{3-\mathrm{CH} 3}\right), 26.3\left(\mathrm{C}_{3^{\prime \prime \prime}}\right), 25.5\left(\mathrm{C}_{2^{\prime \prime}}\right), 25.4$ $\left(\mathrm{C}_{3^{\prime \prime}}\right), 24.1\left(\mathrm{C}_{2^{\prime \prime \prime}}\right), 18.8\left(\mathrm{C}_{6^{\prime}}\right), 18.3\left(\mathrm{C}_{6^{\prime \prime \prime \prime}}\right), 17.3\left(\mathrm{C}_{6^{\prime \prime}}\right)$ and $16.8\left(\mathrm{C}_{6^{\prime \prime \prime}}\right)$.

\section{In vitro SUMOylation assay}

The in vitro SUMOylation reaction was performed for $2 \mathrm{~h}$ at $30^{\circ} \mathrm{C}$ in $20 \mu \mathrm{l}$ buffer (50 mM Tris ( $\mathrm{pH} 7.4$ ), $6 \mathrm{~mm} \mathrm{MgCl}_{2}, 2 \mathrm{~mm}$ ATP and $1 \mathrm{~mm}$ DTT)

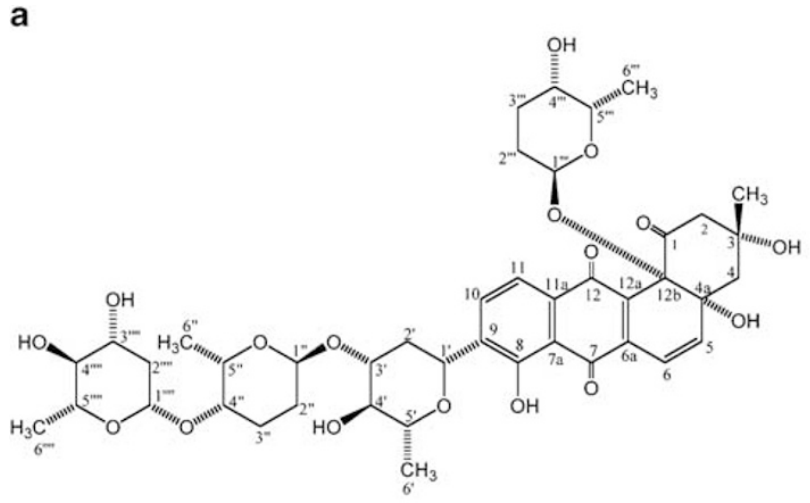

b
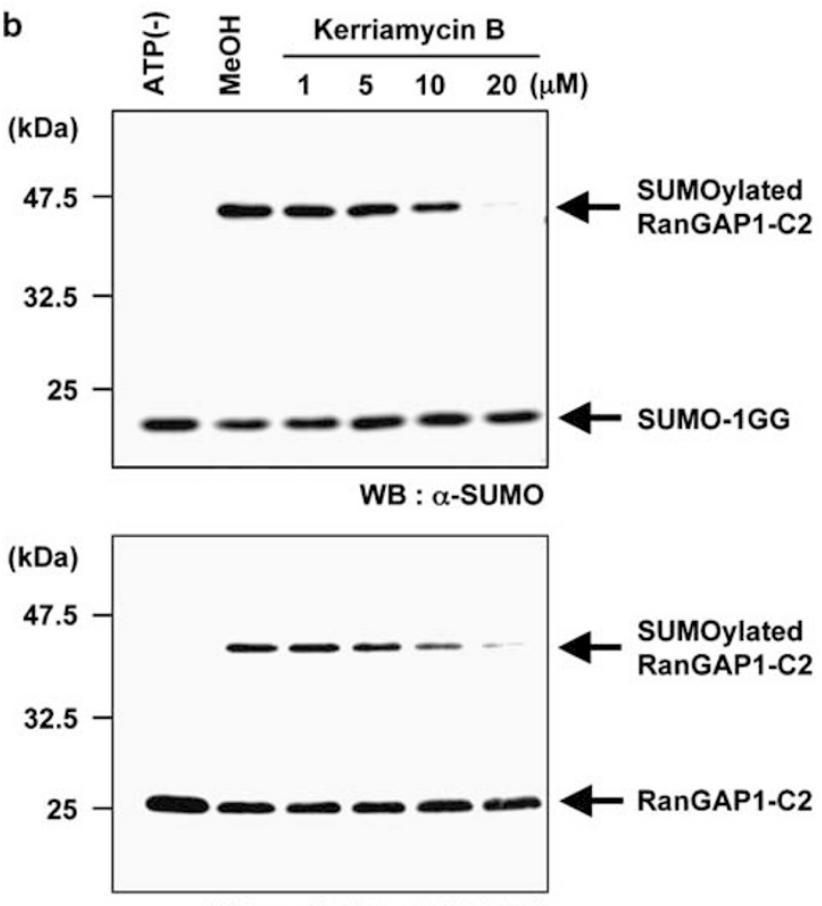

containing $0.1 \mu \mathrm{g}$ of His and T7-tagged RanGAP1-C2, $0.3 \mu \mathrm{g}$ of GST-Aos1/ Uba2 (E1), $0.01 \mu \mathrm{g}$ of His-tagged Ubc9 (E2) and $0.1 \mu \mathrm{g}$ of His-tagged SUMO-1. Samples were separated by $10 \%$ SDS-PAGE followed by immunoblotting using an anti-T7 antibody to detect RanGAP1-C2 or an anti-SUMO-1 antibody.

\section{Assay for SUMO-1 thioester bond formation}

The reaction for the thioester bond formation was performed for $20 \mathrm{~min}$ at $37^{\circ} \mathrm{C}$ in $20 \mu \mathrm{l}$ buffer ( $50 \mathrm{~mm}$ Tris ( $\mathrm{pH} 7.4$ ), $6 \mathrm{~mm} \mathrm{MgCl}_{2}, 2 \mathrm{~mm}$ ATP) containing $1 \mu \mathrm{g}$ of purified GST-Aos1/Uba2 (E1) and $0.1 \mu \mathrm{g}$ of biotinylated SUMO-1 in the absence of DTT. The reaction was stopped by adding loading buffer without the reducing agent. Reaction products were separated by $11 \%$ SDS-PAGE and the E1-biotinylated SUMO-1 intermediate was detected by using avidinconjugated horseradish peroxidase (Sigma).
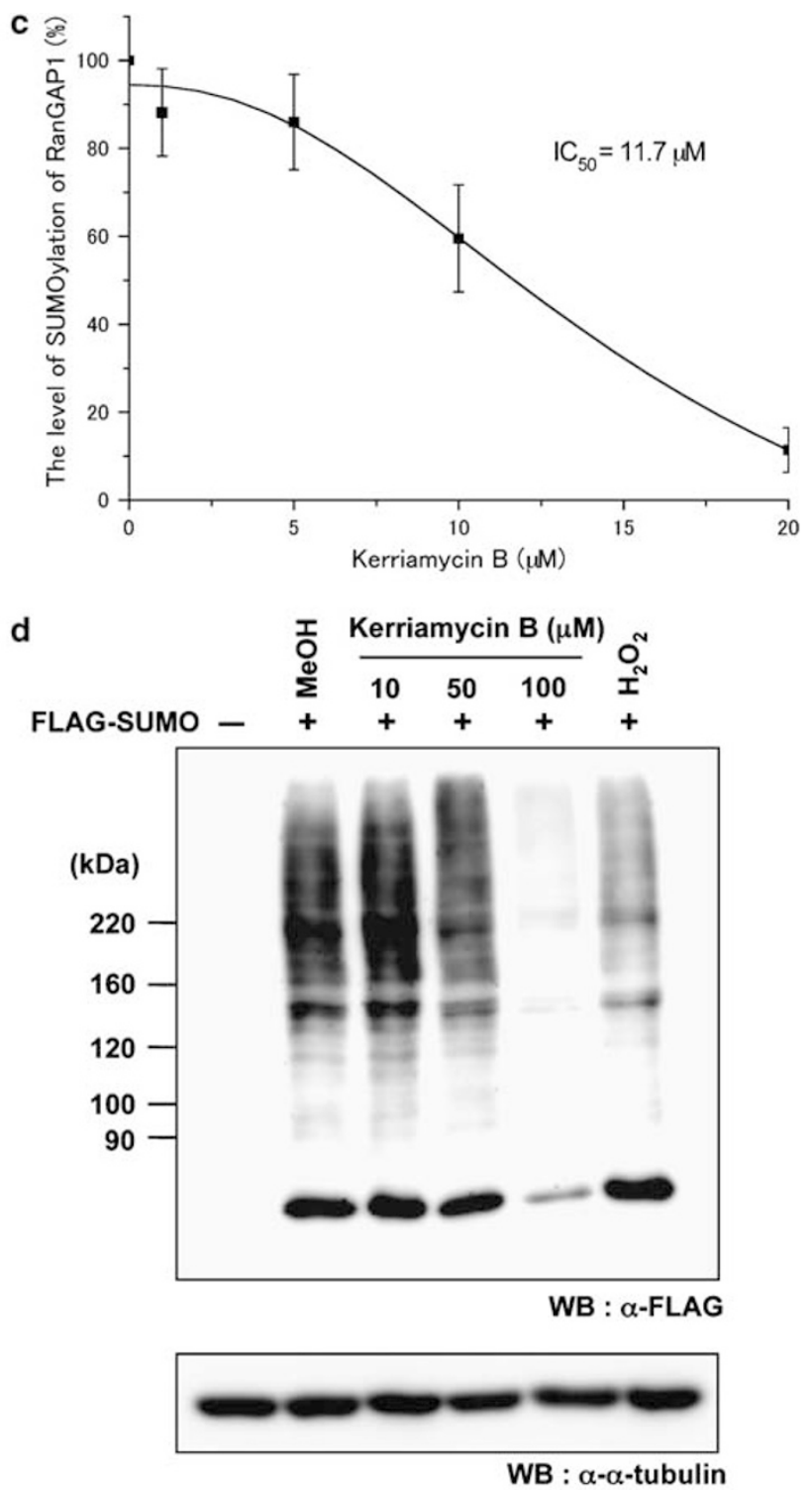

Figure 1 Kerriamycin B inhibition of protein SUMOylation. (a) Structure of kerriamycin B. (b) Dose response of kerriamycin B for SUMOylation inhibition. Indicated concentrations of kerriamycin B (1-20 $\mathrm{m}$ ) were added to the SUMOylation reaction mixture containing His-tagged SUMO-1, His and T7-tagged RanGAP1-C2, the GST-Aos1-Uba2 fusion protein (E1), His-tagged-Ubc9 (E2) in the presence of 2 mM ATP. SUMOlylated RanGAP1-C2 was detected by immunoblotting using an anti-T7 or an anti-SUMO-1 antibody. (c) IC 50 value of kerriamycin B. The level of in vitro SUMOylation of RanGAP1-C2 was determined by measuring the intensity of SUMOylated RanGAP1-C2 using Image Gauge Version 4.22 (FUJIFILM). The error bars show the s.d. from three independent assays and the $\mathrm{IC}_{50}$ value was calculated. (d) Inhibition of in vivo protein SUMOylation by kerriamycin B. 293T cells were transfected with Flagtagged SUMO and then treated with various concentrations of kerriamycin B (10-100 $\mu \mathrm{m})$ for $12 \mathrm{~h}$ or treated with $1 \mathrm{mM} \mathrm{H}_{2} \mathrm{O}_{2}$ for $1 \mathrm{~h}$. Cells were lysed in RIPA buffer containing $50 \mathrm{~mm} \mathrm{~N}$-ethylmaleimide, and the lysates were separated by $6 \%$ SDS-PAGE followed by immunoblotting using an anti-FLAG antibody. 
Using an in situ cell-based SUMOylation assay method, ${ }^{9}$ we screened 1,839 samples of microbial cultured broth, and found extracts from three actinomycete strains showing activity to inhibit protein SUMOylation. We extracted the active substance with EtOAc from one of the extracts, and the active component was separated by using various adsorption column chromatographies. Finally, the pure compound was obtained by reverse phase HPLC. Physico-chemical properties and analysis of NMR and mass spectra showed that this compound was identical with a known antibiotic kerriamycin B (Figure 1a). ${ }^{10}$

To quantitatively analyze the SUMOylation inhibitory activity of kerriamycin B, we first characterized the effect of kerriamycin B on the in vitro protein SUMOylation using RanGAP1-C2 as a substrate. Kerrriamycin B completely inhibited SUMOylation of RanGAP1-C2 in vitro at $20 \mu \mathrm{M}$ (Figure $1 \mathrm{~b}$ ) but not in vitro ubiquitinylation (data not shown). The $\mathrm{IC}_{50}$ value of kerriamycin $\mathrm{B}$ against SUMOylation of RanGAP1-C2 was determined to be $11.7 \mu \mathrm{M}$ (Figure 1c). We then asked whether kerriamycin B also inhibits in vivo protein SUMOylation by analyzing the effect of the level of protein SUMOylation in 293T cells expressing Flag-tagged SUMO (Figure 1d). Immunoblotting using an anti-Flag antibody showed that kerriamycin B reduced the amount of high-molecular weight SUMO conjugates at $100 \mu \mathrm{M}$. Treatment with hydrogen peroxide also reduced the level of high-molecular weight SUMO conjugates (Figure 1d) as recently reported. ${ }^{11}$

Finally, we sought to determine the target of kerriamycin B. The complex of E1 with biotinylated SUMO-1 through the thioester bond can be detected in the presence of ATP under non-reducing conditions using a biotin-avidin detection system. ${ }^{8}$ The band corresponding to the E1-biotinylated SUMO-1 intermediate was detected after incubating E1 with biotinylated SUMO-1 in the presence of ATP, but this band disappeared after addition of the reducing agent DTT (Figure 2). The formation of the E1-biotinylated SUMO-1 intermediate was blocked by kerriamycin B at $20 \mu \mathrm{M}$ (Figure 2). These results suggest that kerriamycin B inhibits protein SUMOylation by blocking the formation of the E1-SUMO-1 intermediate.

Most recently, we identified ginkgolic acid and its related compound anacardic acid present in the plant extract as the first small molecule inhibitors of protein SUMOylation. ${ }^{12}$ Binding assays using a fluorescently labeled ginkgolic acid revealed that ginkgolic acid inhibited protein SUMOylation by directly binding to E1 to block the formation of the E1-SUMO intermediate. In this study, we rediscovered kerriamycin B as a novel inhibitor of protein SUMOylation from microbial metabolites, which also inhibited the formation of the E1-SUMO intermediate. These observations suggested that E1 is the common target for these structurally unrelated compounds.

In addition to its antibacterial activity, kerriamycin B has been shown to possess antitumor activity against Ehrlich ascites carcinoma. ${ }^{10}$ However, the mechanism underlying the antitumor activity is totally unknown. Involvement of the aberrant SUMO system in tumorigenesis has recently been suggested. The increased expression of $u b c 9$ encoding SUMO E2 was reported in several human ovarian cancer cell lines, such as PA- 1 and OVCAR- 8 as well as in ovarian tumor tissues, ${ }^{13,14}$ human lung adenocarcinomas, ${ }^{15}$ and LNCaP metastastic prostate cancer cell line. ${ }^{16}$ These observations might reflect a possible role of Ubc9 in tumorigenesis by regulating SUMOylation of various cellular targets. Therefore, it seems possible that kerriamycin B activity to inhibit SUMOylation is responsible, at least in part, for its antitumor activity. Further analyses on the mechanisms of SUMOylation inhibition and structure-activity relationship of kerriamycin B are necessary for developing a novel anticancer agent targeting aberrant protein SUMOylation.

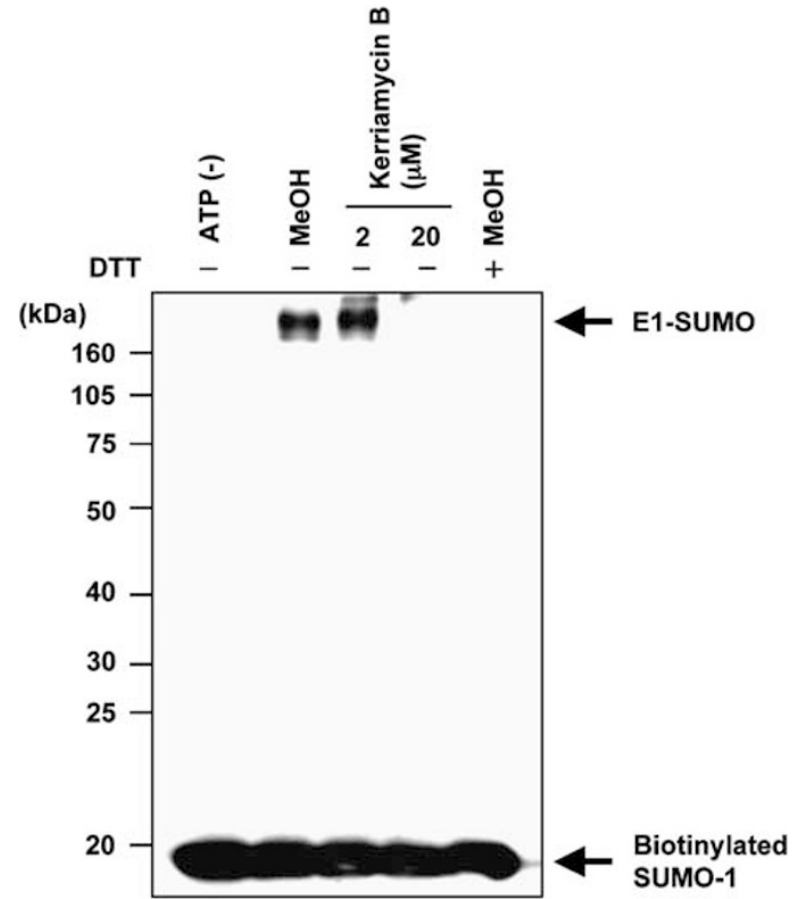

Figure 2 Impairment of the thioester bond formation between E1 and biotinylated SUMO-1 by kerriamycin B. Indicated concentration of kerriamycin B was added to a reaction mixture containing $5 \mathrm{ng} \mathrm{\mu l}^{-1}$ of biotinylated SUMO-1 and $50 \mathrm{ng}^{-1}$ of GST-Aos1/Uba2 in the presence or absence of $2 \mathrm{~mm}$ ATP. After the mixtures had been incubated at $37^{\circ} \mathrm{C}$ for $20 \mathrm{~min}$, they were separated by SDS-PAGE, followed by analysis using avidin-conjugated horseradish peroxidase. Addition of $1 \mathrm{~mm}$ DTT to the reaction completely abolished the complex formation of biotinylated SUMO-1 and GST-Aos1/Uba2.

\section{ACKNOWLEDGEMENTS}

We thank Dr Y Uchimura (Kumamoto University) for technical advice. Microbial cultured broth was supplied from the Broth Screening Network (BSN). This work was supported in part by the Chemical Genomics Research Project, RIKEN ASI, the CREST Research Project, the Japan Science and Technology Corporation, and a Grant-in-Aid for Scientific Research on Priority Area 'Cancer' from the Ministry of Education, Culture, Sports, Science and Technology of Japan.

1 Johnson, E. S. Protein modification by SUMO. Annu. Rev. Biochem. 73, 355-382 (2004).

2 Hay, R. T. SUMO: a history of modification. Mol. Cell 18, 1-12 (2005).

3 Verger, A., Perdomo, J. \& Crossley, M. Modification with SUMO. A role in transcriptional regulation. EMBO Rep. 4, 137-142 (2003).

4 Dorval, V. \& Fraser, P. E SUMO on the road to neurodegeneration. Biochim. Biophys. Acta 1773, 694-706 (2007).

5 Boggio, R. \& Chiocca, S. Viruses and sumoylation: recent highlights. Curr. Opin. Microbiol. 9, 430-436 (2006).

6 Alarcon-Vargas, D. \& Ronai, Z. SUMO in cancer-wrestlers wanted. Cancer Biol. Ther 1, 237-242 (2002).

7 Moschos, S. J. \& Mo, Y. Y. Role of SUMO/Ubc9 in DNA damage repair and tumorigenesis. J. Mol. Histol. 37, 309-319 (2006).

8 Uchimura, Y., Nakao, M. \& Saitoh, H. Generation of SUMO-1 modified proteins in E. coli: towards understanding the biochemistry/structural biology of the SUMO-1 pathway. FEBS Lett. 564, 85-90 (2004).

9 Saitoh, N. et al In situ SUMOylation analysis reveals a modulatory role of RanBP2 in the nuclear rim and PML bodies. Exp. Cell Res. 312, 1418-1430 (2006).

10 Hayakawa, Y. et al Kerriamycins A B and C, New Isotetracenone Antibiotics. Agric. Biol. Chem. 51, 1397-1405 (1987). 
11 Bossis, G. \& Melchior, F. Regulation of SUMOylation by reversible oxidation of SUMO conjugating enzymes. Mol. Cel/ 21, 349-357 (2006).

12 Fukuda, I. et al Ginkgolic acid inhibits protein SUMOylation by blocking formation of the E1-SUMO intermediate. Chem. Biol. (in press).

13 Mo, Y. Y. \& Moschos, S. J. Targeting Ubc9 for cancer therapy. Expert. Opin. Ther. Targets 9, 1203-1216 (2005).
14 Mo, Y. Y. et al A role for Ubc9 in tumorigenesis. Oncogene 24, 2677-2683 (2005). 15 Doniels-Silvers, A. L., Nimri, C. F., Stoner, G. D., Lubet, R. A. \& You, M. Differential gene expression in human lung adenocarcinomas and squamous cell carcinomas. Clin. Cancer Res. 8, 1127-1138 (2002).

$16 \mathrm{Kim}, \mathrm{J}$. H. et al Roles of sumoylation of a reptin chromatin-remodelling complex in cancer metastasis. Nat. Cell Biol. 8, 631-639 (2006). 